\title{
Megakaryocyte Proliferation
}

National Cancer Institute

\section{Source}

National Cancer Institute. Megakaryocyte Proliferation. NCI Thesaurus. Code C19919.

Megakaryocyte Proliferation involves multiplication or reproduction through cell division

of megakaryocytes, a large cell in the bone marrow with a lobulated nucleus that releases mature blood platelets from its cytoplasm. 